\title{
Propriedades físico-químicas e sensoriais de embutidos fermentados formulados com diferentes proporções de carne suína e de ovelhas de descarte
}

\author{
Physico-chemical and sensorial properties of fermented sausages formulated with different \\ proportions of meat from swine and culling ewes
}

\author{
Poliana François ${ }^{I}$ Cleber Cassol Pires ${ }^{I I}$ Letieri Griebler ${ }^{\mathrm{III}}$ Ticiana François $^{\mathrm{IV}}$ \\ Vanessa Souza SorianoI Diego Barcelos Galvaniv
}

\section{RESUMO}

Avaliou-se a inclusão de diferentes níveis de carne de ovelhas de descarte (animais Texel $\times$ Ile de France com idade superior a seis anos) em relação à carne suína sobre a qualidade de embutidos fermentados do tipo salame. Os embutidos foram elaborados com 0, 15, 35, 55 ou $75 \%$ de carne da perna, paleta e pescoço das ovelhas, acrescidos de pernil suíno e $10 \%$ de toucinho suíno. A evolução do $\mathrm{pH}$ e da atividade de água $\left(a_{w}\right)$ foi avaliada nos dias zero, três, sete e 14 após o embutimento. Após a maturação dos salames, foram determinadas, ainda, a perda de peso, a qualidade microbiológica e as características sensoriais dos embutidos prontos. A inclusão de carne ovina na formulação não alterou $a$ evolução do $\mathrm{pH}$ e $a_{w}$. Entretanto, influenciou $(P<0,05)$ os valores finais de $\mathrm{pH}, \mathrm{a}_{w}$ e a perda de peso. Os embutidos com carne ovina na formulação apresentaram valores de $\mathrm{pH}$ final inferiores àquele observado para o embutido elaborado somente com carne suína. Este último apresentou $a_{w}$ inferior $e$ maior perda de peso, em comparação ao produto contendo $15 \%$ de carne ovina na formulação. Todas as formulações atenderam a legislação quanto à qualidade microbiológica. $\mathrm{Na}$ análise sensorial, o embutido elaborado com $15 \%$ de carne ovina foi considerado superior ao embutido elaborado somente com carne suína para os aspectos cor, sabor e textura; contudo, não diferiu dos demais embutidos elaborados com carne ovina. Assim, é possível elaborar embutido fermentado com até $75 \%$ de carne de ovelhas de descarte na formulação.

Palavras-chave: análise sensorial, qualidade, maturação, ovelhas de descarte, salame.

\begin{abstract}
The influence of including different proportions of meat from swine and culling ewes on the quality of fermented sausage type salami was evaluated. Five different formulations were prepared containing $0,15,35,55$, or $75 \%$ of meat from leg, shoulder, and neck of the ewes, added of swine meat and $10 \%$ of swine fat. Evolution of $\mathrm{pH}$ and water activity $\left(a_{w}\right)$ was evaluated on days $0,3,7$, and 14 of the maturation period. After that, it was still determined the weight loss, microbiological quality, and the sensorial features of the sausages. The inclusion of sheep meat in the formulation did not changed $\mathrm{pH}$ and $a_{w}$ evolution. However, differences in final values of $p H, a_{w}$, and weight loss were found $(P<0.05)$. Sausages with sheep meat in the formulation had lower $\mathrm{pH}$ than that without sheep meat. The control sausage (0\% sheep meat) also showed lower $a_{w}$ and a greater weight loss than sausage with $15 \%$ of sheep meat. All formulations attended the actual legislation about microbiological quality. In the sensorial analysis, the sausage prepared with $15 \%$ of sheep meat in the formulation was considered better than that formulated just with swine meat in relation to color, flavor, and texture features. However, there were no differences in sensorial characteristics among sausages with sheep meat. Thus, it is possible to produce fermented sausages with until $75 \%$ of meat from culling ewes in the formulation.
\end{abstract}

Key words: sensorial analysis, quality, maturation, culling sheep, sausage.

\footnotetext{
IPrograma de Pós-graduação em Zootecnia, Universidade Federal de Santa Maria (UFSM), Santa Maria, RS, Brasil.

"IDepartamento de Zootecnia, UFSM. Av. Roraima, 1000, 97105-900, Santa Maria, RS, Brasil. E-mail: cpires@smail.ufsm.br. Autor para correspondência.

IIC Curso de Zootecnia, UFSM, Santa Maria, RS, Brasil.

${ }^{\text {IV }}$ Curso de Agronomia, UFSM, Santa Maria, RS, Brasil.

vPrograma de Pós-graduação em Ciência Animal e Pastagens, Escola Superior de Agricultura Luiz de Queiroz (ESALQ), Universidade de São Paulo (USP), Piracicaba, SP, Brasil.
} 


\section{INTRODUÇÃO}

A ovinocultura brasileira tem apresentado mudanças constantes, principalmente no segmento carne, disponibilizando cortes especiais para redes de supermercados e restaurantes que atendem consumidores de classe média alta. Esse nicho de mercado impulsiona o crescimento da atividade em vários Estados brasileiros, tanto pelo aumento do efetivo do rebanho, quanto pelo incremento do número de empreendimentos rurais destinados à atividade (OJIMA et al., 2006).

O sabor e o aroma da carne ovina são alterados pela idade do animal e pelas condições de criação e manejo. A carne de cordeiro tem sabor suave e cor mais clara, quando comparada com aquela de animais adultos, sendo, portanto, preferida pelos consumidores (KROLOW, 2005). Dessa forma, a oferta de carne de animais adultos no mercado, muitas vezes, compromete a imagem do produto e a consequente efetivação do hábito do consumo.

Uma alternativa de comercialização da carne dessa categoria animal seria por meio da sua industrialização, por exemplo, na forma de embutidos (MATOS et al., 2007; PELEGRINI et al., 2008). A elaboração desse tipo de produto representa uma alternativa viável de processamento, por se tratar de produto estável em temperatura ambiente, o que facilita a comercialização e permite alcançar novos mercados consumidores. Nesse sentido, alguns estudos (KLETTNER et al., 1989; ROÇA, 1993; SCHIFFNER et al., 1996; PELEGRINI et al., 2008) têm demonstrado a viabilidade do uso da carne de ovinos adultos para fabricação de embutidos fermentados e a boa aceitação destes por parte dos consumidores. PELEGRINI et al. (2008) ressaltaram, contudo, a necessidade de realização de estudos utilizando-se carne suína em diferentes proporções, com a finalidade de atingir um melhor nível de aceitabilidade.

Este trabalho foi realizado com objetivo de avaliar o efeito da inclusão de diferentes proporções de carne de ovina, proveniente de animais adultos, em relação à carne suína, sobre os parâmetros de qualidade do embutido fermentado do tipo salame.

\section{MATERIAL E MÉTODOS}

O processamento dos embutidos foi realizado na planta piloto do Laboratório de Carnes do Departamento de Tecnologia e Ciência dos Alimentos da Universidade Federal de Santa Maria (UFSM). As análises físico-químicas, microbiológicas e sensoriais foram realizadas nos laboratórios do Departamento de Tecnologia e Ciência dos Alimentos da UFSM.
A carne ovina utilizada como matéria-prima foi proveniente da desossa da perna, da paleta e do pescoço de ovelhas de descarte, todas com mais de seis anos de idade, oriundas do cruzamento entre as raças Ile de France e Texel. Os animais foram terminados em pastagem cultivada. Visando a caracterizar a matériaprima utilizada, foram determinados os teores de matéria seca, proteína, gordura e matéria mineral da carne ovina (AOAC, 2000). A carne suína utilizada (pernil e toucinho) foi adquirida em estabelecimento comercial local. Foram estabelecidas cinco diferentes proporções entre carne ovina e suína para elaboração dos embutidos: $\mathrm{T}_{0 \%}$ (controle) - embutido fermentado tipo salame formulado com $90 \%$ de carne suína e $10 \%$ de toucinho; $\mathrm{T}_{15 \%}$ embutido fermentado tipo salame formulado com $15 \%$ de carne ovina, $75 \%$ de carne suína e $10 \%$ de toucinho; $\mathrm{T}_{35 \%}$ - embutido fermentado do tipo salame formulado com 35\% de carne ovina, 55\% de carne suína e 10\% de toucinho; $\mathrm{T}_{55 \%}$ - embutido fermentado tipo salame formulado com $55 \%$ de carne ovina, 35\% de carne suína e $10 \%$ de toucinho; $\mathrm{T}_{75 \%}$ - embutido fermentado do tipo salame formulado com $75 \%$ de carne ovina, $15 \%$ de carne suína e $10 \%$ de toucinho.

A trituração das carnes suína e ovina foi realizada utilizando moedor elétrico (Jamar PJ22, Jamar Ltda., São Paulo, Brasil) provido de prato com 8mm de diâmetro e orifícios de $3 \mathrm{~mm}$. Após a moagem, as matérias-primas foram misturadas (Jamar MJI35, Jamar Ltda., São Paulo, Brasil) durante três minutos para a extração das proteínas miofibrilares. Foram adicionados à massa cárnea: cloreto de sódio $\left(30 \mathrm{~g} \cdot \mathrm{kg}^{-1}\right)$, glicose $\left(5 \mathrm{~g} \cdot \mathrm{kg}^{-1}\right)$, sacarose $\left(5 \mathrm{~g} \cdot \mathrm{kg}^{-1}\right)$, pó húngaro II $\left(3 \mathrm{~g} \cdot \mathrm{kg}^{-1}\right)$, pimenta branca $\left(2 \mathrm{~g} \cdot \mathrm{kg}^{-1}\right)$, alho $\left(5 \mathrm{~g} \cdot \mathrm{kg}^{-1}\right)$, noz moscada $\left(0,2 \mathrm{~g} \cdot \mathrm{kg}^{-1}\right)$ e mistura comercial de cura $\left(2,5 \mathrm{~g} \cdot \mathrm{kg}^{-1}\right)$ contendo nitrato e nitrito de sódio (Germinal Aditivos, São Paulo, Brasil), conforme recomendações de TERRA (2005). Ao final, adicionou-se um fixador de cor, e a massa resultante foi misturada por cinco minutos.

O processo fermentativo foi conduzido mediante adição de cultura starter contendo Lactobacillus plantarum e Pediococcus acidilactici (Biobak Sal Plus S3, Wiberg GmbH, Alemanha), diluída em água isenta de cloro 30 minutos antes da adição. A quantidade adicionada à massa foi de $50 \mathrm{~g}$ para cada 100kg de massa cárnea.

Foram elaborados $8 \mathrm{~kg}$ de produto por tratamento, misturados e embutidos separadamente. A massa cárnea foi embutida em tripas naturais de bovinos, previamente mergulhadas em solução de ácido acético, a 2\%, e cortadas em partes com cerca de $15 \mathrm{~cm}$ de comprimento, totalizando 40 peças de aproximadamente $200 \mathrm{~g}$ por tratamento. Três peças de cada tratamento foram pesadas imediatamente após o embutimento para posterior avaliação da perda de peso durante a fermentação (TERRA \& BRUM, 1988). 
As peças foram, então, acondicionadas em câmara climatizada, à temperatura de $25^{\circ} \mathrm{C}$. Essa temperatura foi progressivamente reduzida $\left(1^{\circ} \mathrm{C}\right.$ por dia) até atingir $18^{\circ} \mathrm{C}$. A umidade nos primeiros sete dias de fermentação foi mantida pela presença de um recipiente com água dentro da câmara. No sétimo dia, quando a temperatura já estava em $18^{\circ} \mathrm{C}$, foi retirado o recipiente com água, reduzindo-se, assim, a umidade dentro da câmara, proporcionando o início do processo de maturação. Foi realizado o acompanhamento do pH e da atividade de água $\left(\mathrm{a}_{\mathrm{w}}\right)$ nos dias zero, três, sete e 14 após o embutimento. O salame foi considerado pronto quando a $a_{w}$ atingiu valores inferiores a 0,9 , o que ocorreu no $14^{\mathrm{w}}$ dia após o embutimento.

Para as análises físico-químicas dos embutidos prontos, foram coletados aleatoriamente três gomos de amostra de cada tratamento, sendo as análises realizadas em triplicata. $\mathrm{O} \mathrm{pH}$ foi analisado conforme técnica descrita por TERRA \& BRUM(1998), e a a foi analisada utilizando o equipamento Aqualab CX2T (Decagon Devices, Pullman, Estados Unidos).

As características microbiológicas dos embutidos foram avaliadas no dia da fabricação (dia zero), no dia sete e no produto final, e envolveram a contagem de coliformes a $45^{\circ} \mathrm{C}$, pesquisa de Salmonella e estafilococos coagulase positiva de acordo com SIQUEIRA(1995).

Para avaliação das formulações elaboradas, foi realizada, ainda, a análise sensorial dos salames produzidos. Foram consultados 50 avaliadores, escolhidos ao acaso, apreciadores ou não de salame. Os avaliadores provaram todas as partidas, que estavam identificadas por códigos e compararam estas ao salame elaborado apenas com carne suína (controle). As amostras (duas alíquotas com aproximadamente $10 \mathrm{~g}$ cada, de cada tratamento) foram oferecidas aos avaliadores em pratos plásticos brancos, acompanhadas de um copo de água e biscoito do tipo água e sal. Os aspectos cor, odor, sabor e textura puderam ser classificados como extremamente melhor que o controle (7), muito melhor que o controle (6), regularmente melhor que o controle (5), nenhuma diferença do controle (4), regularmente pior que o controle (3), muito pior que o controle (2) ou extremamente pior que o controle (1) (MORAES, 1988).

O delineamento experimental utilizado foi o inteiramente casualizado, com cinco tratamentos. As médias foram comparadas pelo teste de Tukey, a 5\% de probabilidade do erro Tipo I. Foi também realizada a análise de regressão e, para a análise sensorial, foi utilizado o delineamento de blocos ao acaso, em que cada avaliador foi considerado um bloco. Os dados foram submetidos ao teste de Bonferroni, que comparou os tratamentos com diferentes níveis de substituição da carne suína pela carne ovina com o tratamento controle, que continha somente carne suína. As análises foram feitas utilizando-se o programa estatístico SAS (2001).

\section{RESULTADOS E DISCUSSÃO}

A composição centesimal da carne das ovelhas de descarte utilizada como matéria-prima na elaboração dos embutidos apresentou valores médios de $75,8 \%$ de umidade, $20,8 \%$ de proteína, 3,5\% de gordura e $0,3 \%$ de matéria mineral. PINHEIRO et al. (2007) relataram valor de 20,4\% para o teor de proteína da carne de ovelhas de descarte. Contudo, esses autores observaram maior concentração de gordura na carne $(7,8 \%)$, possivelmente devido ao fornecimento de suplementação concentrada aos animais e uma consequente maior taxa de ganho de peso.

A evolução do $\mathrm{pH}$ e da atividade de água $\left(a_{w}\right)$ em cada tratamento durante o processamento está apresentada na figura 1 . Todos os tratamentos apresentaram o mesmo comportamento, isto é, queda nos valores de $\mathrm{pH}$ e de $\mathrm{a}_{\mathrm{w}}$ no decorrer do tempo. Segundo LÜCKE (1998), o declínio no valor de pH durante os primeiros dias de fermentação é importante para a produção de salames de qualidade devido à inibição de microrganismos indesejáveis, conversão e estabilização da cor e formação de compostos desejáveis, relacionados às características de sabor e aroma. A redução da atividade de água pode ser atribuída à queda dos valores de $\mathrm{pH}$, uma vez que a capacidade de retenção de água das proteínas da carne diminui na medida em que o $\mathrm{pH}$ se aproxima do seu ponto isoelétrico, acelerando a desidratação e consequentemente reduzindo a $\mathrm{a}_{\mathrm{w}}$ (CHASCO et al., 1996).

Na tabela 1, são apresentados os valores médios finais de $\mathrm{pH}, \mathrm{a}_{\mathrm{w}}$ e a quebra ocorrida durante a fermentação e maturação dos embutidos. A incorporação da carne de ovelhas de descarte, juntamente com a carne de suínos na formulação dos embutidos fermentados, influenciou $(\mathrm{P}<0,05)$ os valores finais de $\mathrm{a}_{\mathrm{w}}$ e $\mathrm{pH}$, assim como a perda de peso ocorrida durante a fermentação e maturação dos embutidos. Os valores finais de $a_{w}$, encontrados neste experimento, estão dentro daqueles determinados pela legislação brasileira (BRASIL, 2000), que estabelece o valor máximo de 0,92 para o salame e 0,90 para o salame do tipo italiano.

As formulações contendo carne ovina apresentaram valores finais de $\mathrm{pH}$ inferiores $(\mathrm{P}<0,05)$ ao valor observado para os salames do tratamento 

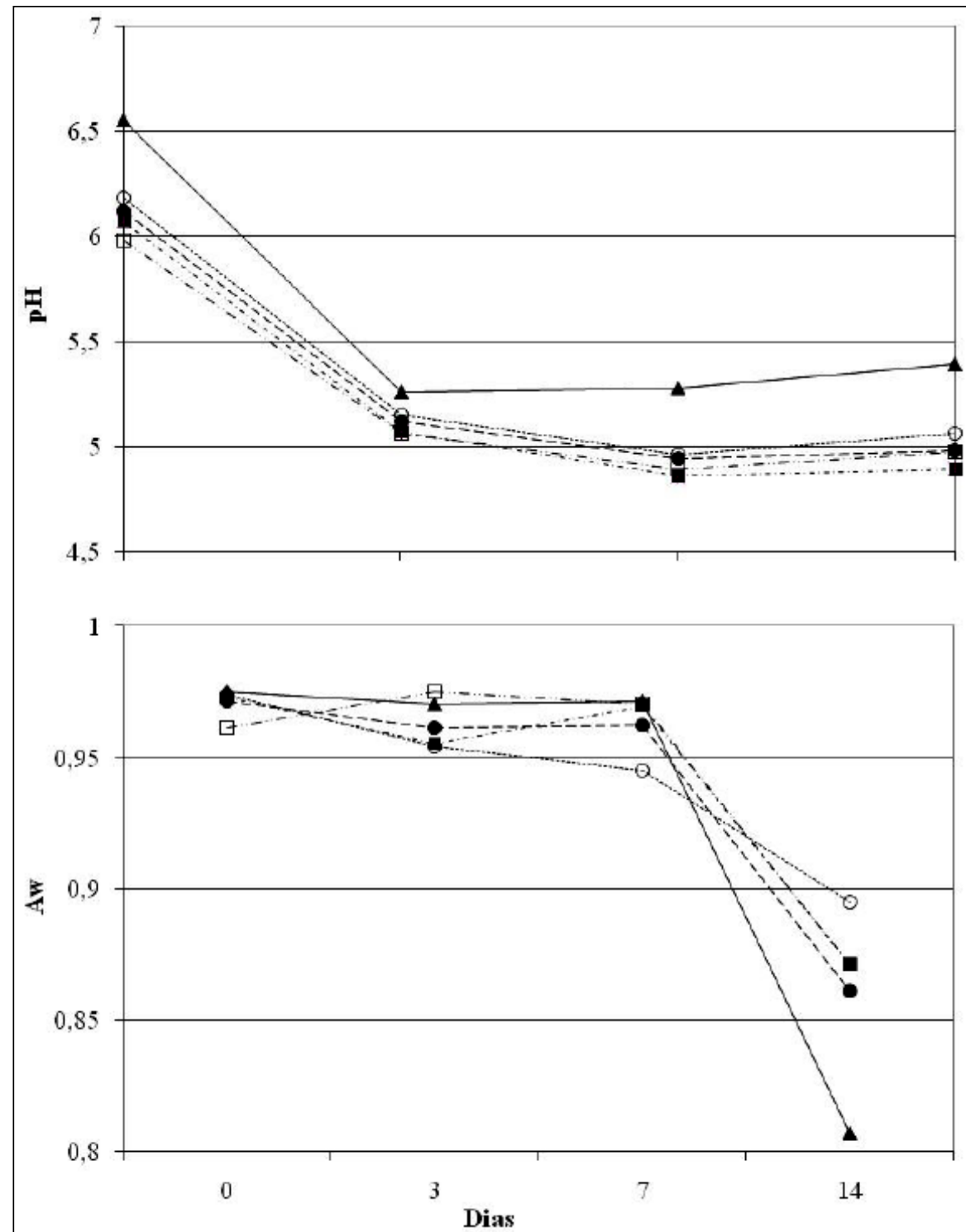

Figura 1 - Evolução do pH e da atividade de água (Aw) dos embutidos fermentados elaborados com 0 ( $\bullet$ ), 15 ( O), 35 ( •), 55 ( $\square$ ) ou 75\% ( 口) de carne de ovelhas de descarte na formulação.

controle. Esse fato, possivelmente, está relacionado ao maior valor de $\mathrm{pH}$ observado para os embutidos produzidos apenas com carne suína também no dia 0 , uma vez que o pH inicial da carne tem influência direta no $\mathrm{pH}$ do produto final. Carnes com $\mathrm{pH}$ mais elevados necessitam maior produção de ácido para alcançar o mesmo $\mathrm{pH}$ final (BACUS, 1984).

A atividade de água é um importante fator determinante do crescimento de microrganismos. A ação inibidora do crescimento é potencializada pelo decréscimo do pH e da adição de cloreto de sódio aos embutidos fermentados. Valores elevados de atividade de água, entre 0,98 e 1, possibilitam o desenvolvimento de diversos tipos de microrganismos, em especial as bactérias. Níveis menores que 0,87 inibem o desenvolvimento da maioria das bactérias e leveduras (MOSSEL \& GARCIA, 1985).

Os embutidos produzidos somente com carne suína (controle) apresentaram a inferior e maior perda de peso que aqueles produzidos com $15 \%$ de carne ovina. RUST (1994) considerou como ideal para produtos fermentados secos uma perda de peso variando de 30 a $40 \%$, o que ocorreu somente com os embutidos contendo $15 \%$ de carne ovina na formulação. Contudo, os resultados deste estudo são semelhantes aos obtidos por REIS \& SOARES (1998), que elaboraram salame colonial de carne suína e carne ovina obtendo valores de perda de peso entre 38,4 e $43,7 \%$. 
Tabela 1 - Valores médios de pH final, atividade de água $\left(\mathrm{a}_{\mathrm{w}}\right)$ e perda de peso após 14 dias de fermentação e maturação dos embutidos formulados com diferentes proporções de carne de ovelhas de descarte.

\begin{tabular}{|c|c|c|c|c|c|c|}
\hline & \multicolumn{5}{|c|}{ 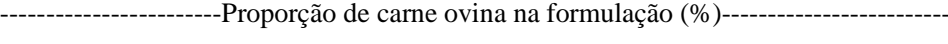 } & \multirow{2}{*}{ EPM* $^{*}$} \\
\hline & 0 & 15 & 35 & 55 & 75 & \\
\hline $\mathrm{pH}$ & $5,39^{\mathrm{a}}$ & $5,07^{\mathrm{b}}$ & $4,98^{\mathrm{C}}$ & $4,89^{d}$ & $4,97^{\mathrm{c}}$ & 0,0052 \\
\hline$a_{w}$ & $0,807^{\mathrm{b}}$ & $0,895^{\mathrm{a}}$ & $0,861^{\mathrm{ab}}$ & $0,870^{\mathrm{ab}}$ & $0,852^{\mathrm{b}}$ & 0,0051 \\
\hline Quebra (\%) & $46,2^{\mathrm{a}}$ & $37,3^{\mathrm{b}}$ & $41,6^{\mathrm{ab}}$ & $43,7^{\mathrm{a}}$ & $41,9^{\mathrm{ab}}$ & 0,46 \\
\hline
\end{tabular}

*erro padrão da média.

Valores seguidos por letras minúsculas diferentes na mesma linha diferem pelo teste de Tukey $(\mathrm{P}<0,05)$.

A qualidade microbiológica dos embutidos foi considerada satisfatória em todas as etapas avaliadas neste experimento. Os valores encontrados para a contagem de bactérias estão em conformidade com a legislação (BRASIL, 2000). Esses resultados podem ser atribuídos à qualidade da matéria-prima utilizada, às condições higiênicas de preparo e às características do próprio produto que possui valor baixo de $\mathrm{pH}$, presença de aditivos, como o nitrito, e baixa atividade de água, o que torna as condições desfavoráveis para o desenvolvimento da maioria dos microrganismos (NASSU et al., 2001).

$\mathrm{Na}$ tabela 2, estão apresentados os resultados obtidos na avaliação sensorial. O embutido fermentado elaborado com $15 \%$ de carne ovina na formulação apresentou coloração pouco melhor $(\mathrm{P}<0,05)$ que aquele elaborado exclusivamente com carne suína. Os demais embutidos não diferiram do controle $(\mathrm{P}<0,05)$ em relação à coloração.

Além disso, segundo a avaliação sensorial, não houve diferenças $(P>0,05)$ entre os embutidos elaborados com carne de ovinos em relação ao elaborado somente com carne suína, quanto ao parâmetro odor. Possivelmente, o pH ácido proporcionado pela fermentação láctica contribuiu para mascarar o aroma característico da carne de ovinos. Além disso, a utilização de culturas starter na elaboração de embutidos fermentados, além do controle de microrganismos deteriorantes e patogênicos, auxilia na melhora do sabor, do aroma e da textura dos produtos (NASSU et al., 2002).

O sabor do embutido elaborado com $15 \%$ de carne ovina na formulação foi considerado $(\mathrm{P}<0,05)$ superior ao embutido elaborado somente com carne suína. No entanto, o sabor desse produto não diferiu $(\mathrm{P}>0,05)$ dos demais embutidos formulados com maior proporção de carne ovina. FERRARI et al. (2001) elaboraram linguiça com até $75 \%$ de carne de ovelhas adultas e observaram boa aceitação do produto pelos consumidores. Também PELEGRINI et al. (2008) elaboraram embutido fermentado do tipo salame utilizando $80 \%$ de carne de ovelhas de descarte e $20 \%$ de pernil suíno na formulação, concluindo que os embutidos são bem aceitos por pessoas consumidoras de salame. Resultado semelhante foi obtido por REIS \& SOARES (1998), que elaboraram salame colonial processado com carne suína e ovina na proporção de 70:30, respectivamente, e concluíram que a elaboração desse tipo de salame é tecnologicamente viável e apresenta boa aceitabilidade pelo público consumidor.

De uma maneira geral, na avaliação sensorial, os embutidos fermentados formulados com diferentes proporções de carne ovina apresentaram resultados satisfatórios, uma vez que, em nenhum aspecto avaliado (cor, odor, sabor e textura), foram obtidos conceitos inferiores ao embutido elaborado somente com carne suína e toucinho.

Tabela 2 - Valores médios de cor, odor, sabor e textura obtidos por meio de análise sensorial dos embutidos elaborados com diferentes proporções de carne de ovelhas de descarte.

\begin{tabular}{|c|c|c|c|c|c|c|}
\hline & \multicolumn{5}{|c|}{------------------------Proporção de carne ovina na formulação (\%)------------------------ } & \multirow{2}{*}{ EPM* } \\
\hline & 0 & 15 & 35 & 55 & 75 & \\
\hline Cor & $4,00^{\mathrm{bc}}$ & $4,92^{\mathrm{a}}$ & $4,62^{\mathrm{ab}}$ & $4,14^{\mathrm{bc}}$ & $3,92^{\mathrm{c}}$ & 0,070 \\
\hline Odor & $4,00^{\mathrm{a}}$ & $4,30^{\mathrm{a}}$ & $4,06^{\mathrm{a}}$ & $4,40^{\mathrm{a}}$ & $4,26^{\mathrm{a}}$ & 0,058 \\
\hline Sabor & $4,00^{\mathrm{b}}$ & $4,76^{\mathrm{a}}$ & $4,28^{\mathrm{ab}}$ & $4,52^{\mathrm{ab}}$ & $4,14^{\mathrm{ab}}$ & 0,072 \\
\hline Textura & $4,00^{\mathrm{b}}$ & $4,74^{\mathrm{a}}$ & $4,54^{\mathrm{ab}}$ & $4,64^{\mathrm{a}}$ & $4,38^{\mathrm{ab}}$ & 0,066 \\
\hline
\end{tabular}

*erro padrão da média.

Valores seguidos por letras minúsculas diferentes na mesma linha diferem estatisticamente pelo teste de Bonferroni (P<0,05).

Ciência Rural, v.39, n.9, dez, 2009. 


\section{CONCLUSÕES}

A inclusão de 55\% de carne de ovelhas de descarte na formulação favorece a obtenção de salames com menor valor de $\mathrm{pH}$ e atividade de água semelhante àquela de embutidos com diferentes proporções de carne ovina e suína após 14 dias de maturação. Além disso, a inclusão de $15 \%$ de carne de ovelhas de descarte na formulação melhora as características de cor, sabor e textura do salame, em comparação aquele produzido somente com carne suína. Níveis maiores de inclusão, contudo, não prejudicam nenhuma das características sensoriais dos salames, sendo, dessa forma, possível a adição de até 75\% de carne de ovelhas de descarte na formulação desse tipo de embutido fermentado.

\section{AGRADECIMENTO}

Cleber Cassol Pires é pesquisador do Conselho Nacional de Desenvolvimento Científico e Tecnológico (CNPq).

\section{REFERÊNCIAS}

ASSOCIATION OF OFFICIAL ANALYTICAL CHEMISTS AOAC. Official Methods of Analysis of AOAC International. 17ed. Gaithersburg, 2000. 1170p.

BACUS, J. Update: meat fermentation. Food Technology, v.38, n.6, p.59-69, 1984

BRASIL. Ministério da Agricultura. Secretaria de Defesa Agropecuária. Instrução Normativa n. 22, de 31 de julho de 2000. Regulamento técnico de identidade e qualidade de salame. Publicada no Diário Oficial da União de 03/08/00.

CHASCO, J. et al. Cured colour development during sausage processing. Meat Science, v.44, n.3, p.203-211, 1996. Disponível em: <http:/ /dx.doi.org/10.1016/S0309-1740(96)00092-7>. Acesso em: 20 mar. 2009. doi: 10.1016/S0309-1740(96)00092-7.

FERRARI, R.A. et al. Composição físico-química e avaliação sensorial de lingüiça de ovelha. In: SIMPÓSIO LATINO AMERICANO DE CIÊNCIA DE ALIMENTOS, 4., 2001, Campinas, SP. Anais... Campinas: R. Vieira, 2001. p.176-176.

KLETTNER, P.G. et al. Processing of old sheep in the meat industry. Fleischwirtschaft, v.69, n.12, p.1810-1835, 1989.

KROLOW, A.C.R. Qualidade do alimento x perspectiva de consumo da carne ovina e caprina. 2005. Disponível em: <http://www.spmv.org.br/conpavet2004/palestras\%20$\% 20$ resumos/palestra_Ana\%20Cristina\%20Krolov.doc $>$. Acesso: nov de 2008.

LÜCKE, F.K. Fermented sausages. In: WOOD, B.J.B (Ed.). Microbiology of fermented foods. 2.ed. London: Blackie Academy Professional, 1998. p.441-483.

MATOS, R.A. et al. Efeito do tipo de fermentação na qualidade final de embutidos fermentados cozidos elaborados a base de carne ovina. Boletim do Centro de Pesquisa e Processamento de Alimentos, v.25, n.2, p.225-234, 2007. Disponível em: <http://ojs.c3sl.ufpr.br:80/ojs2/index.php/ alimentos/article/view/10610>. Acesso em: nov de 2008.

MORAES, M.A.C. Métodos para avaliação sensorial dos alimentos. 6.ed. Campinas: UNICAMP, 1988. 93p.

MOSSEL, D.A.A.; GARCIA, B.M. Microbiologia de los alimentos. Zaragoza: Acríbia, 1985. 375p.

NASSU, R.T. et al. Estudo das características físico-químicas, microbiológicas e sensoriais de embutidos fermentados tipo salame formulados com diferentes proporções de carne caprina e suína. Boletim do Centro de Pesquisa e Processamento de Alimentos, v.19, n.2, p.243-256, 2001. Disponível em: <http://ojs.c3sl.ufpr.br:80/ojs2/index.php/alimentos/article/ view/1236>. Acesso em: nov de 2008.

NASSU, R.T. et al. Utilização de diferentes culturas starter no processamento de embutido fermentado de carne de caprinos. Ciência Rural, v.32, n.6, p.1051-1055, 2002. Disponível em: $<$ http://dx.doi.org/10.1590/S0103-84782002000600021>. Acesso em: 20 mar. 2009. doi: 10.1590/S0103-84782002000600021.

OJIMA, A.L.R.O. et al. Caprinos e ovinos em São Paulo atraem argentinos. Análise e Indicadores do Agronegócio, v.1, n.1, 2006. Disponível em: <http://www.iea.sp.gov.br/out/ verTexto.php?codTexto=4462>. Acesso: nov. de 2008 .

PELEGRINI, L.F.V. et al. Elaboração de embutido fermentado tipo salame utilizando carne de ovelhas de descarte. Ciência e Tecnologia de Alimentos, v.28, n.Supl., p.150-153, 2008. Disponível em: <http://dx.doi.org/10.1590/S0101-20612008000500023>. Acesso em: 20 mar. 2009. doi: 10.1590/S0101-20612008000500023.

REIS, A.G.B.; SOARES, G.J.D. Salame colonial processado com carne suína e ovina. Revista Brasileira de Agrociência, v.2, n.2, p.115-120, 1998.

PINHEIRO, R.S.B. et al. Informações nutricionais de carnes ovinas em rótulos comerciais, comparativamente às obtidas em análises laboratoriais. Ciência e Tecnologia de Alimentos, v.27, n.2, p.375-380, 2007. Disponível em: <http://dx.doi.org/10.1590/S0101-20612007000200028>. Acesso em: 20 mar. 2009. doi: 10.1590/S010120612007000200028.

ROÇA, R.O. Alternativas de aproveitamento da carne ovina. Revista Nacional da Carne, n.201, p.53-60, 1993.

RUST, R.E. Productos embutidos. In: PRICE, J.F., SCHWEIGERT, B.S. Ciencia de la carne y de productos cárnicos. 2.ed. Zaragoza: Acríbia, 1994. p.415-440.

SCHIFFNER, E. et al. Elaboración casera de carne y embutidos. Zaragoza: Acribia, 1996. 298p.

SIQUEIRA, S. Manual de microbiologia de alimentos. Brasília: EMBRAPA, 1995. 159p.

STATISTICAL ANALYSIS SYSTEM - SAS. System for Microsoft Windows: release 8.2. Cary: 2001. CD-ROM.

TERRA, N.N.; BRUM, M.A.R. Carne e seus derivados técnicas de controle de qualidade. São Paulo: Nobel, 1988. $121 \mathrm{p}$.

TERRA, N.N. Apontamentos de tecnologia de carnes. São Leopoldo: Unissinos, 2005. 216p. 